\title{
Transition metals redox: reviving an old plot for diabetic vascular disease
}

\author{
Vincent M. Monnier \\ Case Western Reserve University, Institute of Pathology, Cleveland, Ohio 44106, USA. \\ Phone: (216) 368-6613; Fax: (216) 368-0495; E-mail: vmm3@po.cwru.edu.
}

\begin{abstract}
Considerable effort over the past 25 years has focused on the role of oxidant stress in aging and in the pathogenesis of age-related diseases - diabetes, Alzheimer's disease, end-stage renal disease, and atherosclerosis, among others. Research on redox signaling and the chemistry of the aging process has led to major insights, including the identification of oxidant stress-responsive transcription factors, such as NF- $\mathrm{kB}$, which regulate tissue remodeling and therefore control the progression of pathological lesions; the role of mitochondria in generating reactive oxygen species and activating apoptotic pathways; the role of sulfhydryl homeostasis in redox signaling; and the development of mass spectrometry methods to identify and quantify protein damage in aging or stressed tissues.
\end{abstract}

Because of the prevalence and the dire consequences of the diseases involved, the stakes in this field are high. However, despite the great interest in developing drugs that might block oxidant or carbonyl stress, clinical studies involving antioxidant or carbonyl-trapping agents have had mixed success, suggesting a greater degree of complexity than anticipated. Thus, in the diabetic rat, treatment with various antioxidants or carbonyl-trapping agents has had impressive effects in delaying, if not altogether preventing, complications of diabetes such as cataracts, retinopathy, nephropathy, vascular abnormalities, nerve conduction velocity, plasma lipid oxidation, and fetal malformations. In the diabetic human, conversely, while intra-arterial infusion of vitamin $\mathrm{C}$ improved endothelium-dependent vasodilation (1) and oral intake of vitamin E improved retinal blood flow and creatinine clearance (2), chronic treatment with vitamin $\mathrm{E}$ did not reduce cardiovascular risk (3). Similarly, the antioxidant $\alpha$-lipoic acid decreased plasma hydroperoxides in diabetic subjects but had equivocal efficacy in polyneuropathy and cardiac autonomic neuropathy $(4,5)$. A similar "antioxidant paradox" has also been observed in other diseases associated with oxidant stress (6).

\section{Protein oxidation in diabetes}

In diabetes, the controversy has focused on the origin, the type, the magnitude, and the localization of oxidant stress in relation to hyperglycemia, and how this combination promotes the progression of micro- and macrovascular disease. For example, high glucose levels were associated early on with an altered cellular redox state, aldose reductase activation, and impaired glutathione homeostasis in selected tissues. In experimental diabetes, antioxidants or transition metal chelators can ameliorate retinopathy and neuropathy, suggesting that oxidant stress contributes to this condition $(7,8)$. On the other hand, Williamson et al., noting an increased cellular NADH/NAD ratio (9), have proposed that diabetes is a state of "reductive" stress and "pseudohypoxia," raising the question of how oxidative damage might arise in a reducing environment.

As the glycation theory of diabetic complications unfolded, metal-catalyzed glucose autooxidation and oxidation of glycated residues emerged as potent sources of free radicals and were proposed as the primary culprits in tissue damage $(10,11)$. In vitro, exposing proteins to high levels of glucose causes oxidative protein fragmentation and damage to amino acid residues, with the accumulation of methionine sulfoxide, $o$-tyrosine, $m$-tyrosine, and other modifications. Many of the glucose-associated oxidative modifications have been attributed to Fenton chemistry carried out by transition metals like copper and iron, which are normally present in phosphate buffer (11). Wolff et al. and Baynes therefore proposed a key role for oxidation and glycoxidation chemistry in the pathogenesis of diabetic complications $(10,11)$. More recently, however, the model of generalized oxidant stress lost support because of a lack of evidence for increased levels of oxidized skin collagen in diabetic individuals (reviewed in ref. 12). Instead, Baynes and Thorpe propose a greater role for overload of metabolic pathways as the primary culprit in oxidant and carbonyl stress in diabetes: "Treatment of diabetes with antioxidant therapy," they write, "is like applying water to a burning house, certainly helpful in limiting the conflagration, but also a little bit late in the process" (12).

Oxidative damage in atherosclerosis research has also focused investigators on the effects of transition metals. $\mathrm{Cu}^{2+}$-catalyzed LDL oxidation has become a useful and widely studied, albeit controversial, model for oxidative events in the arterial wall. Coincubation of LDL with glucose or glycated proteins significantly increases lipoprotein oxidation by adventitious transition metals, thus offering a potential explanation for the acceleration of atherosclerosis in diabetes (13). In this context, the useful terms "glycoxidation" and "lipoxidation" have found their way in the literature (12).

However, another potentially relevant mechanism of oxidation emerged with the exciting discovery that myeloperoxidase, derived from macrophages of the arterial wall, potently oxidizes LDL to generate the same oxidative modifications found in LDL isolated from atheromatous plaques. Both $\mathrm{Cu}^{2+}$ mediated and myeloperoxidase-mediated oxidation lead to an increase in $o$ tyrosine and $m$-tyrosine, but only the latter selectively generates dityrosine from tyrosine radical (reviewed in ref. 14). The finding that dityrosine was selectively increased in fatty streaks and intermediate atheromatous lesions, whereas hydroxyl radical damage was elevated only in more advanced lesions, 


\section{Figure 1}

Proposed sequence of events leading to hydroxyl radical-mediated protein damage in early atherosclerosis in diabetes. The data from Pennathur et al. (16) show a strong relationship between hydroxyl radical damage and hemoglobin glycation. Because these authors found no evidence for increased nitration-mediated damage, it appears that formation of the initial lesion does not involve inflammatory cells. A likely scenario involves increased glycation and the formation of the redox-active center due to the formation of carboxymethyl-lysine $(\mathrm{CML})$ and carboxyethyl-lysine (CEL), which can bind redox-active copper and perhaps iron. Amadori products and ceruloplasmin (not shown) are also expected to be potent precursors of oxidative damage. Hyperglycemia-catalyzed superoxide formation from mitochondrial and cytoplasmic sources is expected to initiate the lipoxidation cascade and release of glyoxal, a potent CML precursor. PUFA, polyunsaturated fatty acid.

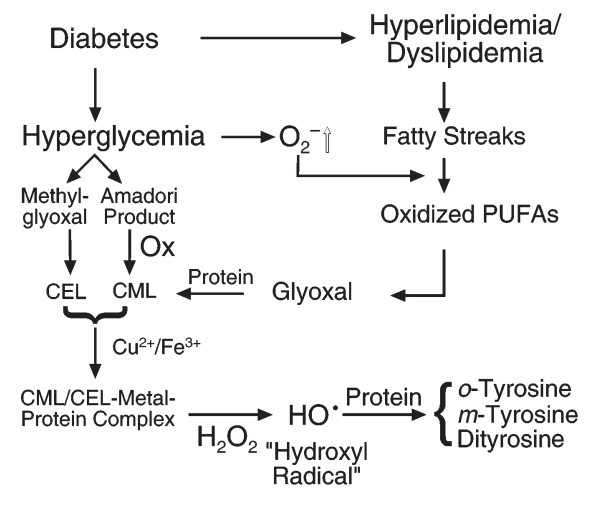

mechanism for the protein damage reported by Pennathur et al. (16). Of major interest in this context is the recent suggestion of Saxena et al. (23) that ascorbic acid, which is also found in atheromatous plaques, can generate CML and become a pro-oxidant in the presence of transition metals.

Still unclear is the exact source of the transition metals. Possibilities include the transfer of loosely bound metals to CML/CEL-rich proteins, which could result from glycation of superoxide dismutase, ceruloplasmin, or ferritin (24), and the possible binding of redox-active iron by Amadori products (25). However, intact ceruloplasmin can also oxidize lipoproteins (26), and its levels are increased in selected patients with diabetes.

The apparent absence of myeloperoxidase- and nitration-mediated oxidation suggests that inflammatory cells are not involved at the very early stage of atherogenesis in diabetes. This scenario may be specific for diabetes, since previous data from apparently nondiabetic individuals suggest the contrary (14). However, once they become oxidized and accumulate CML and other advanced glycation products, vesselassociated LDL and other proteins can act as signals and chemotactic factors for activation of inflammatory cells by binding to RAGE, CD36, or other receptors (27-29). Once that barrier has been crossed, it is not surprising that many forms of protein damage ensue.

If the mechanisms put forward in Figure 1 apply to the early phase of atherosclerosis in diabetes, then therapeutic antioxidants will be needed much earlier in the process than previously appreciated. Transition metal-chelating agents and hydroxyl radical scavengers may prove useful as adjuvants to other forms of therapy. 
1. Ting, H.H., et al. 1996. Vitamin C improves endothelium-dependent vasodilation in patients with non-insulin-dependent diabetes mellitus. J. Clin. Invest. 97:22-28.

2. Bursell, S.E., et al. 1999. High-dose vitamin E supplementation normalizes retinal blood flow and creatinine clearance in patients with type 1 diabetes. Diabetes Care. 22:1245-1251.

3. Hoogwerf, B.J., and Young, J.B. 2000. The HOPE study. Ramipril lowered cardiovascular risk, but vitamin E did not. Cleve. Clin. J. Med. 67:287-293.

4. Reljanovic, M., et al. 1999. Treatment of diabetic polyneuropathy with the antioxidant thioctic acid (alpha-lipoic acid): a two year multicenter randomized double-blind placebo-controlled trial (ALADIN II). Alpha Lipoic Acid in Diabetic Neuropathy. Free Radic. Res. 31:171-179.

5.Ziegler, D., et al. 1999. Treatment of symptomatic diabetic polyneuropathy with the antioxidant alpha-lipoic acid: a 7-month multicenter randomized controlled trial (ALADIN III Study). ALADIN III Study Group. Alpha-Lipoic Acid in Diabetic Neuropathy. Diabetes Care. 22:1296-301.

6. Halliwell, B. 2000. The antioxidant paradox. Lancet. 355:1179-1180.

7. Cameron, N.E., and Cotter, M.A. 1995. Neurovascular dysfunction in diabetic rats. Potential contribution of autoxidation and free radicals examined using transition metal chelating agents. J. Clin. Invest. 96:1159-1163.

8. Kunisaki, M., et al. 1995. Vitamin E prevents diabetes-induced abnormal retinal blood flow via the diacylglycerol-protein kinase $\mathrm{C}$ pathway. Am. J. Physiol. 269:E239-E246.

9. Williamson, J.R., et al. 1993. Perspectives in diabetes: hyperglycemic pseudohypoxia and diabetic complications. Diabetes. 42:801-813.

10. Wolff, S.P., Jiang, Z.Y., and Hunt, J.V. 1991. Protein glycation and oxidative stress in diabetes mellitus and ageing. Free Radic. Biol. Med. 10:339-352.

11. Baynes, J.W. 1991. Role of oxidative stress in development of complications in diabetes. Diabetes. 40:405-412.

12. Baynes, J.W., and Thorpe, S.R. 1999. Role of oxidative stress in diabetic complications: a new perspective on an old paradigm. Diabetes. 48:1-9.

13. Mullarkey, C.J., Edelstein, D., and Brownlee, M. 1990. Free radical generation by early glycation products: a mechanism for accelerated atherogenesis in diabetes. Biochem. Biophys. Res. Commun. 173:932-939.

14. Heinecke, J.W. 1998. Oxidants and antioxidants in the pathogenesis of atherosclerosis: implications for the oxidized low density lipoprotein hypothesis. Atherosclerosis. 141:1-15.

15. Semenkovich, C., and Heinecke, J.W. 1997. The mystery of diabetes and atherosclerosis: time for a new plot. Diabetes. 46:327-334.

16. Pennathur, S., Wagner, J.D., Leeuwenburgh, C. Litwak, K.N., and Heinecke, J.W. 2001. A hydroxyl radical-like species oxidizes cynomolgus monkey artery wall proteins in early diabetic vascular disease. 107:853-860.

17. Baynes, J.W., and Thorpe, S.R. 2000. Glycoxidation and lipoxidation in atherogenesis. Free Radic. Biol. Med. 28:1708-1716.

18. Nishikawa, T., et al. 2000. Normalizing mitochondrial superoxide production blocks three pathways of hyperglycaemic damage. Nature 404:787-790.

19. Ellis, E.A., et al. 1998. Increased NADH oxidase activity in the retina of the BBZ/Wor diabetic rat. Free Radic. Biol. Med. 24:111-120.

20. Imanaga, Y., et al. 2000. In vivo and in vitro evi dence for the glycoxidation of low density lipoprotein in human atherosclerotic plaques. Atherosclerosis. 150:343-355.

21. Nagai, R., et al. 1997. Hydroxyl radical mediates
$\mathrm{Ne}$-(carboxymethyl)lysine formation from Amadori product. Biochem. Biophys. Res. Commun. 234:167-172.

22. Saxena, A.K., et al. 1999. Protein aging by carboxymethylation of lysines generates redox active and divalent metal binding sites: rele vance to diseases of glycoxidative stress. Biochem. Biophys. Res. Commun. 260:332-338.

23. Saxena, P., et al. 2000. Transition metal-catalyzed oxidation of ascorbate in human cataract extracts: possible role of advanced glycation end products. Invest. Ophthalmol. Vis. Sci. 41:1473-1481

24. Taniguchi, N., et al. 1995. Glycation of metalcontaining proteins such as $\mathrm{Cu}, \mathrm{Zn}$-superoxide dismutase, ceruloplasmin, and ferritin: possible implication for DNA damage in vivo. Contrib. Nephrol. 112:18-23.

25. Qian, M., Liu, M., and Eaton, J.W. 1998. Transition metals bind to glycated proteins forming redox active "glycochelates": implications for the pathogenesis of certain diabetic complications. Biochem. Biophys. Res. Commun. 250:385-389.

26. Fox, P.L., et al. 2000. Ceruloplasmin and cardiovascular disease. Free Radic. Biol. Med. 28:1735-1744.

27. Ohgami, N., et al. 2001. CD36, a member of class B scavenger receptor family, as a receptor for advanced glycation end products (AGE). J. Biol. Chem. 276:3195-3202.

28. Schmidt, A.M., et al. 1999. Activation of receptor for advanced glycation end products: mechanism for chronic vascular dysfunction in diabetic vasculopathy and atherosclerosis. Circ. Res. 84:489-497.

29. Stitt, A.W., et al. 2000. Advanced glycation endproduct receptor interactions on microvascular cells occur within caveolin-rich membrane domains. FASEB J. 14:2390-2392. 